\title{
Conhecimento de Universitários da Área da Saúde sobre o Câncer de Cavidade Oral
}

doi: https://doi.org/10.32635/2176-9745.RBC.2019v65n2. 97

\author{
University Health Knowledge on Oral Cavity Cancer \\ Conocimiento de Universitarios del Área de la Salud sobre el Cáncer de Cavidad Oral
}

\author{
Carina Balem Ganze'; Gabriela Wagner²; Ariana Machado Toriy³; Suellen Cristina Roussenq ${ }^{4}$; Cristiana Pezzi Franco de Souza5; \\ Grasiani Breggue Pires; ${ }^{6}$ Mirella Dias ${ }^{7}$
}

Resumo

Introduçáo: $\mathrm{O}$ câncer de cabeça e pescoço engloba um grupo heterogêneo de neoplasias malignas originadas na cavidade oral, faringe, laringe, seios paranasais, cavidade nasal, glândulas salivares e tireoide. Objetivo: Este estudo buscou avaliar o conhecimento dos universitários da área da saúde sobre o câncer de cavidade oral. Método: Estudo do tipo epidemiológico, transversal, descritivo, quali-quantitativo com amostra não probabilística de conveniência. Os universitários receberam, via formulário on-line, um questionário com 24 questōes aplicadas que abordavam os fatores de risco, sintomatologia, aspectos clínicos, autoexame, prevenção e tratamento. Resultados: Participaram do estudo 110 universitários da área da saúde $(58$ = primeiro ano e 52 = último ano), distribuídos nos cursos de fisioterapia, odontologia, psicologia, educação física, enfermagem, medicina e nutrição. Em relação à média de acertos dos questionários, os universitários do primeiro ano obtiveram $77,65 \%( \pm 21,09)$ de acertos, e os universitários do último ano, $82,37 \%( \pm 19,88)$. As questôes que obtiveram menores taxas de acertos foram as relacionadas à incidência do câncer de boca no Brasil; 43 (39\%) acertaram e 52 (47,3\%) dos universitários desconhecem a idade em que maioria dos casos são diagnosticados. Conclusáo: Apesar de o câncer de cavidade oral ser crescente, formas de prevenção e diagnóstico precoce reservam maiores chances de cura. A partir deste estudo, pôde-se concluir que os universitários dos cursos da área da saúde, principalmente do último ano, parecem estar aptos a informar à populaçáo a respeito do câncer de cavidade oral. Palavra-chave: Neoplasias; Neoplasias de Cabeça e Pescoço; Neoplasias Bucais; Desempenho Acadêmico; Epidemiologia.

\section{Abstract}

Introduction: Head and neck cancer comprises a heterogeneous group of malignant neoplasms originating in the oral cavity, pharynx, larynx, paranasal sinuses, nasal cavity, salivary and thyroid glands. Objective: This study aimed to evaluate the knowledge of university students in the field of oral cancer. Method: A cross-sectional, descriptive, qualitative-quantitative study with a non-probabilistic convenience sample. The students received an online questionnaire with 24 applied questions that addressed the risk factors, symptomatology, clinical aspects, self-examination, prevention and treatment. Results: 110 university students from the health area $(58=$ first year and 52 = last year) participated in the study, distributed in physical therapy, dentistry, psychology, physical education, nursing, medicine and nutrition. Regarding the average number of correct answers to the questionnaires, the first-year students had an average of $77.65 \%( \pm 21.09)$ of correct answers, and the last year's college students $82.37 \%( \pm 19.88)$. The questions that obtained the lowest rates of correct answers were the ones related to the incidence of oral cancer in Brazil, where 43 (39\%) were correct, and $52(47.3 \%)$ of the academics are unaware of the age at which most cases are diagnosed. Conclusion: Although cancer of the oral cavity is increasing, forms of prevention and early diagnosis reserve greater chances of cure. From this study, we can conclude that the academics of health courses, especially in the last year, seem to be able to inform the population about cancer of the oral cavity.

Key words: Neoplasms; Head and Neck Neoplasms; Mouth Neoplasms; Academic Performance; Epidemiology.
Resumen

Introducción: El câncer de cabeza y cuello compreende um grupo heterogéneo de neoplasias malgnas que se originan em la cavidade oral. Objetivo: Este estudio buscó evaluar el conocimiento de los universitarios del área de la salud sobre el cáncer de cavidad oral. Método: Estudio del tipo epidemiológico, transversal, descriptivo, cual-cuantitativo con muestra no probabilística de conveniencia. Los académicos recibieron, a través del formulario en línea, un cuestionario con 24 preguntas aplicadas que abordaban los factores de riesgo, sintomatología, aspectos clínicos, autoexamen, prevención y tratamiento. Resultados: Participaron del estudio 110 universitarios del área de la salud (58 = primer año y 52 = último ańo), distribuidos en los cursos de fisioterapia, odontología, psicología, educación física, enfermería, medicina y nutrición. En cuanto a la media de aciertos de los cuestionarios, los universitarios del primer año obtuvieron una media del $77,65 \%( \pm 21,09)$ de aciertos, y los universitarios del último año $82,37 \%$ ( \pm $19,88)$. Las cuestiones que obtuvieron menores tasas de aciertos fueron las relacionadas a la incidencia del cáncer de boca en Brasil; 43 (39\%) acertaron y $52(47,3 \%)$ de los académicos desconocen la edad en la cual la mayoría de los casos son diagnosticado. Conclusión: A pesar de que el cáncer de cavidad oral es creciente, formas de prevención y diagnóstico precoz reservan mayores posibilidades de curación. A partir de este estudio se pode concluir que los académicos de los cursos del área de la salud, principalmente del último año, parecen estar aptos para informar a la población acerca del cáncer de cavidad oral.

Palabras clave: Neoplasias; Neoplasias de Cabeza y Cuello; Neoplasias de la Boca; Rendimiento Académico; Epidemiología.

\footnotetext{
${ }^{1}$ Universidade do Sul de Santa Catarina (Unisul). Florianópolis (SC), Brasil. Orcid iD: https://orcid.org/0000-0001-6003-7065

${ }^{2}$ Unisul. Florianópolis (SC), Brasil. Orcid iD: https://orcid.org/0000-0002-0967-1463

${ }^{3}$ Universidade do Estado de Santa Catarina (Udesc). Florianópolis (SC), Brasil. Orcid iD: https://orcid.org/0000-0002-0372-0711

${ }^{4}$ Udesc. Centro de Pesquisas Oncológicas (Cepon). Florianópolis (SC), Brasil. Orcid iD: https://orcid.org/0000-0001-8202-6244

${ }^{5}$ Cepon. Florianópolis (SC), Brasil. Orcid iD: https://orcid.org/0000-0002-6862-6275

${ }^{6}$ Universidade Nove de Julho (Uninove). São Paulo (SP), Brasil. Orcid iD: https://orcid.org/0000-0002-1683-7054

${ }^{7}$ Unisul. Florianópolis (SC), Brasil. Orcid iD: https://orcid.org/0000-0002-2109-3563
}

Endereço para correspondência: Ariana Machado Toriy. Rua Pascoal Simone, 865 - Coqueiros. Florianópolis (SC), Brasil. CEP 88080-350. E-mail: arianatoriy@gmail.com 


\section{INTRODUÇÃO}

O câncer compreende um conjunto de inúmeros tipos diferentes de doenças que apresentam um crescimento desordenado de células anormais, com potencial invasivo em tecidos e orgãos ${ }^{1}$. O câncer de cabeça e pescoço engloba um grupo heterogêneo de neoplasias malignas originadas na cavidade oral, faringe, laringe, seios paranasais, cavidade nasal, glândulas salivares e tireoide ${ }^{2}$. Entre essa divisão, há predomínio do "câncer oral" que corresponde às mucosas de boca e faringe ${ }^{3}$.

A incidência de câncer de cabeça e pescoço é de 550 mil casos novos com aproximadamente 300 mil mortes anualmente. O câncer de cavidade oral (CCO) ocupa a $15^{\circ}$ colocação entre as neoplasias mais frequentes, com uma incidência de 299.051 novos casos anualmente ${ }^{4}$. De acordo com o Instituto Nacional de Câncer José Alencar Gomes da Silva (INCA) ${ }^{1}$, estimam-se, para cada ano do biênio de 2018/2019, no Brasil, 600 mil novos casos de câncer, sendo 11.200 de CCO em homens e 3.500 em mulheres. E, para Santa Catarina, são previstos 590 novos casos de $\mathrm{CCO}^{1}$.

Topograficamente, o CCO afeta os lábios e o interior da boca, podendo acometer gengivas, mucosa jugal, palato duro, língua e assoalho bucal ${ }^{1}$. A manifestação pode se apresentar por dor na boca, garganta, mandíbula ou ouvido, ferida na boca que não cicatriza, caroço na bochecha ou pescoço, dificuldade para mover a língua, mastigar e engolir, mudança na voz, perda de peso, entre outras. Entre os principais fatores de risco, encontram-se a radiação solar, a contaminação da boca e da garganta pelo papilomavírus humano (HPV) ${ }^{5}$ e o tabagismo e o etilismo, apresentando aumento do fator de risco em 30 vezes quando há associação no consumo ${ }^{6}$.

Em razão dos dados supracitados e da relevância da enfermidade apresentada, o diagnóstico precoce é importante, uma vez que, se tratado adequadamente, $80 \%$ dos casos desse tipo de câncer têm cura ${ }^{1}$. A deficiência de conhecimento na formação profissional tem sido apontada como uma das causas de diagnósticos tardios ${ }^{7}$. Considerando o câncer como um problema de saúde pública ${ }^{8}$, e dada a importância da participação e da contribuição dos profissionais de saúde como disseminadores do conhecimento, torna-se imprescindível conhecer o nível de entendimento a respeito do CCO, tais como prevenção, formas de detecção precoce e tratamento, repercutindo em altas mais eficientes, melhor prognóstico e aumento das taxas de sobrevida dessa populaçãa ${ }^{1,9}$.

Diante desse cenário, o estudo objetivou avaliar o conhecimento dos estudantes da área da saúde de uma universidade do Sul do país a respeito do CCO.

\section{MÉTODO}

Este estudo é do tipo epidemiológico, transversal, descritivo, quali-quantitativo com amostra não probabilística por conveniência.

Após aprovação do Comitê de Ética em Pesquisa da Universidade do Sul de Santa Catarina (Unisul), sob o protocolo $\mathrm{n}^{\mathrm{o}}$. 86288218.9.0000.5369, todos os 388 universitários dos cursos da área da saúde (fisioterapia, nutrição, psicologia, educação física, odontologia, enfermagem e medicina) foram convidados, com aprovação dos coordenadores de cada curso, e mediante e-mail enviado uma única vez, a participar da pesquisa. Esses universitários estavam regularmente matriculados no primeiro e último ano, do primeiro semestre de 2018. Foram excluídos universitários com menos de 18 anos e aqueles que não responderam e/ou responderam indevidamente ao questionário juntamente com o Termo de Consentimento Livre e Esclarecido (TCLE), assinado eletronicamente.

A coleta foi realizada no mês de maio de 2018, por meio da ferramenta Google Forms, utilizando um questionário autoaplicativo que possui 24 questôes fechadas de múltipla escolha. Cada questão possui apenas uma resposta correta, abordando temas específicos acerca do conhecimento do câncer de boca, referente à etiologia, epidemiologia, fatores de risco, sintomatologia, aspectos clínicos, tratamento, prognóstico, autoexame e prevenção. O questionário ficou disponível por 15 dias, juntamente com o aceite eletrônico do TCLE.

De forma concomitante à coleta, os dados foram armazenados em uma planilha do Microsoft Excel $^{\circledR}$ e posterior exportação para o programa de análise estatística Statistical Package for the Social Sciences $\left(\mathrm{SPSS}^{\circledR}\right)$. Foi realizada a análise estatística descritiva com frequência relativa para apresentação dos resultados.

\section{RESULTADOS}

Foram avaliados 110 questionários respondidos adequadamente, do total de 388 estudantes convidados, regularmente matriculados na área da saúde da Unisul (primeiro ano e último ano), sendo eles distribuídos nos cursos de fisioterapia (31), medicina (14), odontologia (14), educação física (6), psicologia (19), nutrição (25) e enfermagem (1), coletados no período de maio de 2018.

Para a apresentação dos resultados, as perguntas e respostas dos questionários foram divididas em relação à etiologia do câncer de boca, epidemiologia, fatores de risco, sintomatologia, autoexame e prevenção e, por fim, tratamento do câncer.

Pode-se observar na Tabela 1 que, quando questionados sobre a existência do câncer de boca, 107 (97,3\%) 
universitários reconhecem sua existência, e três (2,7\%), sendo eles do primeiro ano, responderam não conhecer.

Sobre a definição do câncer de boca, 104 (94,5\%) universitários responderam corretamente ao assinalar que o câncer é uma doença, mas não é transmitida de uma pessoa para outra; apenas seis (5,5\%), sendo cinco universitários do primeiro ano, desconheceram essa afirmação. Não houve dúvidas quanto ao câncer não ser uma doença.
Na questão que abordou as causas do câncer, 98 (89,1%) dos universitários responderam que o câncer ocorre em razão do crescimento desordenados das células, porém 12 (10,9\%) universitários ainda tinham dúvidas sobre sua causa.

$\mathrm{Na}$ Tabela 2, observando os aspectos relacionados à epidemiologia do câncer, 96 (87,3\%) universitários reconhecem que o câncer de pele é o mais frequente em

Tabela 1. Frequência absoluta (n) e frequência relativa (\%) sobre a etiologia do câncer de cavidade oral

\begin{tabular}{|c|c|c|c|c|c|c|}
\hline Tabela de Frequências & N1 & $\% 1$ & N2 & $\% 2$ & $\mathbf{N g}$ & $\% g$ \\
\hline \multicolumn{7}{|l|}{ O câncer de boca: } \\
\hline Não é doença & - & 0 & - & 0 & - & 0 \\
\hline É uma doença, mas não é transmitido de uma pessoa para outra & 53 & 91,3 & 51 & 98,1 & 104 & 94,5 \\
\hline É uma doença e pode ser transmitido de uma pessoa para outra & 5 & 8,7 & 1 & 1,9 & 6 & 5,5 \\
\hline \multicolumn{7}{|l|}{ O câncer ocorre por causa: } \\
\hline Crescimento desordenado das células & 51 & 87,9 & 47 & 90,3 & 98 & 89,1 \\
\hline Morte das células & 2 & 3,4 & 1 & 1,9 & 3 & 2,7 \\
\hline Aumento volumétrico das células & 2 & 3,4 & 1 & 1,9 & 3 & 2,7 \\
\hline Ataque de anticorpos contra corpos estranhos & 2 & 3,4 & 2 & 3,8 & 4 & 3,6 \\
\hline Penetração de vírus nas células & 1 & 1,8 & 1 & 1,9 & 2 & 1,8 \\
\hline
\end{tabular}

Tabela 2. Frequência absoluta (n) e frequência relativa (\%) sobre a epidemiologia do câncer de cavidade oral

\begin{tabular}{|c|c|c|c|c|c|c|}
\hline Tabela de Frequências & N1 & $\% 1$ & N2 & $\% 2$ & Ng & $\% g$ \\
\hline Tabela de frequências & N1 & $\% 1$ & N2 & $\% 2$ & $\mathrm{Ng}$ & $\% g$ \\
\hline \multicolumn{7}{|l|}{$\begin{array}{l}\text { Para você quais dos tipos de câncer ocorrem com mais } \\
\text { frequência em países tropicais? }\end{array}$} \\
\hline De lábio & 1 & 1,8 & 4 & 7,6 & 5 & 4,5 \\
\hline De pele & 51 & 87,9 & 45 & 86,5 & 96 & 87,3 \\
\hline De pescoço & - & 0 & 2 & 3,8 & 2 & 1,8 \\
\hline De pulmão & 6 & 10,2 & 1 & 1,9 & 7 & 6,4 \\
\hline \multicolumn{7}{|l|}{ Para você, o câncer de boca ocorre em maior frequência: } \\
\hline Em homens & 45 & 77,5 & 46 & 88,4 & 91 & 82,7 \\
\hline Em mulheres & 13 & 22,1 & 6 & 11,4 & 19 & 17,3 \\
\hline \multicolumn{7}{|l|}{ Você sabe se existe câncer de boca? } \\
\hline Sim & 55 & 94,8 & 52 & 100 & 107 & 97,3 \\
\hline Não & 3 & 5,2 & - & 0 & 3 & 2,7 \\
\hline \multicolumn{7}{|l|}{ Qual a incidência do câncer de boca no Brasil? } \\
\hline $\begin{array}{l}11 \text { por } 100 \text { mil habitantes entre os homens e } 4 \text { por } 100 \text { mil } \\
\text { habitantes entre as mulheres }\end{array}$ & 20 & 34,4 & 23 & 44,2 & 43 & 39 \\
\hline $\begin{array}{l}4 \text { por } 100 \text { mil habitantes entre os homens e } 11 \text { por } 100 \text { mil } \\
\text { habitantes entre as mulheres }\end{array}$ & 17 & 29,3 & 5 & 9,6 & 22 & 20 \\
\hline $\begin{array}{l}700 \text { por } 100 \text { mil habitantes entre os homens e } 300 \text { por } 100 \text { mil } \\
\text { habitantes entre as mulheres }\end{array}$ & 16 & 27,5 & 19 & 36,5 & 35 & 31,8 \\
\hline $\begin{array}{l}300 \text { por } 100 \text { mil habitantes entre os homens e } 700 \text { por } 100 \text { mil } \\
\text { habitantes entre as mulheres }\end{array}$ & 5 & 8,6 & 5 & 9,6 & 10 & 9,0 \\
\hline \multicolumn{7}{|l|}{$\begin{array}{l}\text { Em que faixa de idade a maioria dos casos de câncer de } \\
\text { boca são diagnosticados? }\end{array}$} \\
\hline Abaixo 20 anos & - & 0 & - & 0 & - & 0 \\
\hline Entre 20 a 30 anos & 10 & 17,2 & 4 & 7,6 & 14 & 12,7 \\
\hline Entre 30 a 40 anos & 22 & 37,9 & 14 & 26,8 & 36 & 32,7 \\
\hline Entre 40 a 60 anos & 23 & 39,5 & 29 & 55,6 & 52 & 47,3 \\
\hline Mais de 60 anos & 3 & 5,1 & 5 & 9,6 & 8 & 7,3 \\
\hline
\end{tabular}


países tropicais, e 14 (12,3\%) ficaram em dúvida entre lábio, pescoço e pulmáo, sendo sete universitários do primeiro e sete do último ano.

Em relação à prevalência do câncer de boca, 91 $(82,7 \%)$ acertaram ao assinalar ser mais comum entre homens, e apenas $52(47,3 \%)$ responderam que a faixa etária mais comum é entre 40 e 60 anos, sendo 29 (55,6\%) assinaladas pelos universitários do último ano. Apenas 43 (39\%) universitários, sendo 23 do último ano, acertaram que a incidência do CCO é de 11 por 100 mil habitantes entre os homens e quatro por 100 mil habitantes entre as mulheres.

A Tabela 3 aponta que 83 (75,5\%) dos universitários estavam cientes de que o fumo é o principal fator de risco para o CCO. Porém, apenas $58(52,7 \%)$ sabiam que o fumo e o álcool são os principais fatores de risco quando associados. Não houve dúvidas quanto ao hábito de fumar ser prejudicial à saúde e que o fumo passivo pode ser também prejudicial. Vale ressaltar que $82(74,5 \%)$ dos universitários responderam que o cigarro possui mais de 1.500 substâncias químicas, 42 das alternativas assinaladas são do último ano.

Sobre o tipo de pele mais comum para aparecimento de câncer de boca e face, 58 (52,7\%) universitários responderam corretamente ao afirmar que a pele muito clara exerce maior influência no aparecimento desse tipo de câncer. Sendo a maioria, 33 (56,8\%), assinalada pelos universitários do primeiro ano.

Na Tabela 4, pode-se observar que $101(91,8 \%)$ universitários souberam responder que no início o câncer

Tabela 3. Frequência absoluta (n) e frequência relativa (\%) sobre os fatores de risco do câncer de cavidade oral

\begin{tabular}{|c|c|c|c|c|c|c|}
\hline Tabela de Frequências & N1 & $\% 1$ & N2 & $\% 2$ & $\mathbf{N g}$ & $\% g$ \\
\hline \multicolumn{7}{|c|}{ Qual é o principal fator de risco para o câncer de boca? } \\
\hline Álcool & 1 & 1,7 & 1 & 1,92 & 2 & 1,8 \\
\hline Exposição solar & 6 & 10,3 & 4 & 7,6 & 10 & 9,1 \\
\hline Fumo & 42 & 72,4 & 41 & 78,7 & 83 & 75,5 \\
\hline Vírus & 2 & 3,4 & 2 & 3,8 & 4 & 3,6 \\
\hline Herança genética & 7 & 12 & 4 & 7,6 & 11 & 1 \\
\hline \multicolumn{7}{|c|}{$\begin{array}{l}\text { Qual a associação entre fatores de risco que aumenta } \\
\text { consideravelmente as chances de ter câncer de boca? }\end{array}$} \\
\hline Não existe associação perigosa & - & 0 & - & 0 & - & 0 \\
\hline Fumo e exposição solar & 21 & 36,1 & 16 & 30,7 & 37 & 33,6 \\
\hline Exposição solar e álcool & 1 & 1,72 & 1 & 1,92 & 2 & 1,8 \\
\hline Fumo e álcool & 31 & 53,3 & 27 & 51,8 & 58 & 52,7 \\
\hline Vírus e fumo & 5 & 8,6 & 8 & 15,3 & 13 & 11,8 \\
\hline Álcool e vírus & 0 & 0 & 0 & 0 & 0 & 0 \\
\hline \multicolumn{7}{|c|}{ Você acha que fumar é prejudicial para sua saúde? } \\
\hline Sim & 58 & 100 & 52 & 100 & 110 & 100 \\
\hline Não & - & 0 & - & 0 & - & 0 \\
\hline \multicolumn{7}{|c|}{$\begin{array}{l}\text { Você acha que fumar perto de outras pessoas pode ser } \\
\text { prejudicial para elas? }\end{array}$} \\
\hline Sim & 58 & 100 & 52 & 100 & 110 & 100 \\
\hline Não & - & 0 & - & 0 & - & 0 \\
\hline \multicolumn{7}{|c|}{ Você acha que existam doses seguras para o uso do cigarro? } \\
\hline Sim & 2 & 3,5 & 4 & 7,7 & 6 & 5,5 \\
\hline Não & 56 & 96,5 & 48 & 92,3 & 104 & 94,5 \\
\hline \multicolumn{7}{|c|}{ Quantas substâncias químicas existem no cigarro? } \\
\hline Nenhuma & 2 & 3,4 & - & 0 & 2 & 1,8 \\
\hline 15 & 2 & 3,4 & 1 & 1,9 & 3 & 2,7 \\
\hline 300 & 14 & 24 & 9 & 17,2 & 23 & 20,9 \\
\hline Mais de 1.500 & 40 & 68,9 & 42 & 80,7 & 82 & 74,5 \\
\hline \multicolumn{7}{|c|}{$\begin{array}{l}\text { Qual tipo de pele você acha que influencia mais no } \\
\text { aparecimento de um câncer de lábio e face? }\end{array}$} \\
\hline Pele muito clara & 33 & 56,8 & 25 & 48,1 & 58 & 52,7 \\
\hline Pele clara & 6 & 10,3 & 9 & 17,2 & 15 & 13,6 \\
\hline Pele escura & 6 & 10,3 & 1 & 1,9 & 7 & 6,4 \\
\hline Qualquer uma das anteriores & 13 & 22,5 & 17 & 32,6 & 30 & 27,3 \\
\hline
\end{tabular}


Tabela 4. Frequência absoluta (n) e frequência relativa (\%) sobre a sintomatologia e aspectos clínicos do câncer de cavidade oral

\begin{tabular}{|c|c|c|c|c|c|c|}
\hline $\begin{array}{l}\text { Tabela de frequências } \\
\end{array}$ & N1 & $\% 1$ & N2 & $\% 2$ & $\mathbf{N g}$ & $\% g$ \\
\hline \multicolumn{7}{|l|}{ No início, o câncer de boca: } \\
\hline Dói muito & 3 & 5,7 & 6 & 11,5 & 9 & 8,2 \\
\hline Não dói & 55 & 94,3 & 46 & 88,4 & 101 & 91,8 \\
\hline \multicolumn{7}{|c|}{$\begin{array}{l}\text { Dos sintomas abaixo, qual ou quais você acha que têm a } \\
\text { ver com câncer de boca? }\end{array}$} \\
\hline Dificuldade de falar & 1 & 1,9 & - & 0 & 1 & 0,9 \\
\hline Dificuldade de mastigar & 5 & 8,6 & 1 & 1,9 & 6 & 5,5 \\
\hline Dificuldade de engolir & 2 & 3,4 & - & 0 & 2 & 1,8 \\
\hline Emagrecimento rápido & - & 0 & 2 & 3,8 & 2 & 1,8 \\
\hline Todas as anteriores & 47 & 80,8 & 45 & 86,4 & 92 & 84,4 \\
\hline Nenhuma das respostas anteriores & 3 & 5,1 & 4 & 7,6 & 7 & 5,5 \\
\hline \multicolumn{7}{|c|}{$\begin{array}{l}\text { Se você percebesse uma alteração há mais de } 15 \text { dias em } \\
\text { sua boca, o que você faria? }\end{array}$} \\
\hline Não me preocuparia com isso & - & 0 & 1 & 1,9 & 1 & 0,9 \\
\hline Aplicaria algum medicamento por conta própria & 3 & 5,1 & 3 & 5,7 & 6 & 5,5 \\
\hline Aguardaria mais tempo para ver se desapareceria & 7 & 12,2 & 6 & 11,5 & 13 & 11,8 \\
\hline Iria à benzedeira & 2 & 3,4 & - & 0 & 2 & 1,8 \\
\hline Procuraria um médico ou dentista & 46 & 79,2 & 42 & 80 & 88 & 80 \\
\hline
\end{tabular}

de boca não dói e apenas seis $(11,5 \%)$ do último ano disseram que a doença provoca dor.

A respeito das complicaçôes que podem surgir sobre esse tipo de câncer, $92(84,4 \%)$ dos universitários assinalaram que a doença pode implicar em dificuldade na fala, ao mastigar, ao engolir, e o emagrecimento rápido faz parte dos sintomas da doença. Em contrapartida, seis $(5,5 \%)$ deles concordaram que nenhuma dessas respostas seriam corretas.

Quando questionados sobre a percepção de uma alteração na boca há mais de 15 dias, 88 (80\%) dos universitários procurariam um médico ou dentista para solução do problema, seis $(5,5 \%)$ aplicariam algum medicamento, $13(11,8 \%)$ aguardariam mais um tempo, dois $(1,8 \%)$ procurariam uma benzedeira e apenas um $(0,9 \%)$ não se preocuparia com alteraçóes há mais de duas semanas.

Com relação à prevenção do câncer de boca, dez $(9,1 \%)$ universitários ainda acham que a alimentação não influencia. Entretanto, 100 (90,9\%) deles sabem que os alimentos apropriados para a prevenção são frutas e vegetais. Ter uma alimentação saudável, não fumar, não beber e proteger-se contra o sol são maneiras de prevenir o câncer de boca, e essa afirmativa foi assinalada por 106 $(96,4 \%)$ universitários.

É importante ressaltar que 76 (69,1\%) dos universitários desconhecem o autoexame de boca; destes, 42 (72,5\%) são do primeiro ano. Para realizar o autoexame, são necessários um espelho e um ambiente bem iluminado, e $90(81,8 \%)$ dos universitários concordaram com essa afirmação. Apenas 11 (10\%) universitários disseram que para o autoexame é necessário apenas alguém ajudar. Os outros nove $(8,2 \%)$ universitários, sendo oito $(13,7 \%)$ do primeiro ano, assinalaram que é necessário algum instrumento de dentista para a realizaçáo do autoexame.

$\mathrm{Na}$ Tabela 5, quando questionados a respeito do tratamento do câncer de boca, 109 (99,1\%) universitários reconheceram que o câncer tem cura, e 93 (84,5\%) sabiam que a cirurgia, a radioterapia e a quimioterapia são métodos de tratamento para esse tipo de câncer.

Por fim, em relação à média de acertos do questionário sobre o conhecimento do CCO, os universitários do primeiro ano dos cursos da área da saúde obtiveram uma média aritmética de 77,65 $( \pm 21,09)$ de acertos, e os universitários do último ano, 82,37 $( \pm 19,88)$.

\section{DISCUSSÃO}

A proposta do estudo foi verificar o conhecimento dos universitários do primeiro e último ano da área da saúde a respeito do CCO. Dessa forma, os resultados apontam que poucos universitários não têm conhecimento sobre a existência do $\mathrm{CCO}$ e, apesar de ser um número baixo, ainda assim, indicam a necessidade de maior explanação a respeito desse tipo de câncer, em virtude de sua incidência crescente. Já com relação aos aspectos epidemiológicos e fatores de risco associados, os universitários demonstram haver uma lacuna na compreensão da doença. De acordo com Goldemberg et al. ${ }^{9}$, os indicadores epidemiológicos do CCO estáo relacionados desde a falta de educação até o diagnóstico tardio da doença. Portanto, os profissionais de saúde devem estar a par das evidências epidemiológicas 
Tabela 5. Frequência absoluta (n) e frequência relativa (\%) sobre o autoexame e prevenção do câncer de cavidade oral

\begin{tabular}{|c|c|c|c|c|c|c|}
\hline $\begin{array}{l}\text { Tabela de frequências } \\
\end{array}$ & N1 & $\% 1$ & N2 & $\% 2$ & $\mathbf{N g}$ & $\% g$ \\
\hline \multicolumn{7}{|l|}{$\begin{array}{l}\text { Você acha que sua alimentação pode ajudar na } \\
\text { prevenção do câncer de boca? }\end{array}$} \\
\hline Sim & 51 & 87,9 & 49 & 94,2 & 100 & 90,9 \\
\hline Não & 7 & 12,1 & 3 & 5,8 & 10 & 9,1 \\
\hline \multicolumn{7}{|l|}{$\begin{array}{l}\text { Quais tipos de alimentos podem ajudar na prevenção do } \\
\text { câncer de boca? }\end{array}$} \\
\hline Nenhum & & & & & 8 & 7,3 \\
\hline Frutas e vegetais & 51 & 87,9 & 49 & 94,2 & 100 & 90,9 \\
\hline Carne vermelha & - & 0 & - & 0 & - & 0 \\
\hline Pães e massas & - & 0 & - & 0 & - & 0 \\
\hline Gordura & 1 & 1,72 & 1 & 1,92 & 2 & 1,8 \\
\hline \multicolumn{7}{|l|}{ Você sabe o que é autoexame da boca? } \\
\hline Sim & 16 & 27,5 & 19 & 36,5 & 34 & 30,9 \\
\hline Não & 42 & 72,5 & 33 & 63,5 & 76 & 69,1 \\
\hline \multicolumn{7}{|l|}{ O que é necessário para fazer o autoexame de boca? } \\
\hline Algum instrumento de dentista & 8 & 13,7 & 1,92 & 1 & 9 & 8,2 \\
\hline Somente espelho e um ambiente bem iluminado & 45 & 77,5 & 45 & 86,5 & 90 & 81,8 \\
\hline Ter alguém para ajudar & 5 & 8,6 & 6 & 11,5 & 11 & 10 \\
\hline \multicolumn{7}{|l|}{ O que é preciso fazer para prevenir o câncer de boca? } \\
\hline Ter uma alimentação saudável & - & 0 & - & 0 & - & 0 \\
\hline Não fumar & 2 & 0 & 1 & 1,92 & 3 & 2,7 \\
\hline Não beber & - & 0 & - & 0 & - & 0 \\
\hline Proteger-se contra o sol & 1 & 1,72 & - & 0 & 1 & 0,9 \\
\hline Todas as anteriores & 54 & 93,1 & 52 & 100 & 106 & 96,4 \\
\hline Nenhuma das anteriores & - & 0 & - & 0 & - & 0 \\
\hline \multicolumn{7}{|l|}{ Para você, o câncer de boca: } \\
\hline Não tem cura, vou sofrer sempre & 1 & 1,8 & 0 & 0 & 1 & 0,9 \\
\hline Tem cura, mas preciso estar atento e fazer acompanhamento & 57 & 98,2 & 52 & 100 & 109 & 99,1 \\
\hline Tem cura e posso esquecer desse problema para sempre & - & 0 & - & 0 & - & 0 \\
\hline \multicolumn{7}{|l|}{$\begin{array}{l}\text { Você já ouviu falar de algum dos métodos empregados } \\
\text { para o tratamento de um câncer? }\end{array}$} \\
\hline Cirurgia & 4 & 6,8 & - & 0 & 4 & 3,6 \\
\hline Radioterapia & 1 & 1,72 & - & 0 & 1 & 0,9 \\
\hline Quimioterapia & 5 & 8,6 & 2 & 3,8 & 7 & 6,4 \\
\hline Todos acima & 43 & 74,1 & 50 & 96,1 & 93 & 84,5 \\
\hline Psiquiatria & - & 0 & - & 0 & - & 0 \\
\hline Nenhum & 5 & 8,6 & - & 0 & 5 & 4,5 \\
\hline
\end{tabular}

e dos fatores de risco associados ao CCO para que possam educar a populaçáo e seus pacientes quanto às questóes relacionadas a esse câncer ${ }^{10}$, que ocupa o quinto lugar de incidência entre todos os tipos de câncer em homens e o sétimo em mulheres ${ }^{7}$.

Em um estudo mais recente, Chen et al. ${ }^{11}$ relatam que o CCO é o oitavo tipo de câncer mais comum no mundo. Ainda que essa prevalência comparada com outras neoplasias não seja alta, na China, foi relatado que existem 21.413 novos casos e 11.333 mortes pelo câncer em $2012^{11,12}$. Nos países nórdicos, foi realizado um estudo de coorte que comparou o risco de câncer de boca e faringe em diferentes ocupaçóes, com dados coletados de 14,9 milhóes de pessoas, sendo encontrados 28.623 casos de câncer de língua, cavidade oral e faringe. Na maioria dos casos, as ocupaçôes consideradas de alto risco diminuíram seus índices e as ocupaçôes de baixo risco aumentaram seus índices, após o ajuste para o uso de tabaco e álcool, conforme o esperado ${ }^{13}$

Em relação aos fatores de risco, nos homens acima de 50 anos, houve um aumento significativo de CCO relacionado ao HPV; enquanto, para as mulheres, a incidência permaneceu estável ${ }^{14}$. Nesse quesito, na presente pesquisa, os universitários apontaram o CCO como sendo o mais comum em homens, provavelmente por estarem mais expostos a fatores de risco conforme 
ressalta Moro et al. ${ }^{15}$. Importante salientar que, nas próximas décadas, o fator de risco em pacientes mais velhos com maior exponencial de crescimento associado ao CCO será o HPV ${ }^{16}$. Chotipanich et al. ${ }^{17}$ ainda sugerem que fumar pode agravar os cânceres relacionados ao HPV, mas que o vírus, até então, tem menor influência nas neoplasias do que outros fatores de risco, como o tabagismo.

A maioria dos universitários considerou o fumo como o principal fator de risco para o câncer de boca e sua associação com o álcool. Bezerra et al. ${ }^{18}$ ressaltaram um pior prognóstico quando o CCO tem como fatores de risco essa associação. Embora o tabaco e álcool continuem sendo os fatores de risco predominantes para o $\mathrm{CCO}^{11,19,20}$, o estudo de Domingos et al. ${ }^{7}$ teve uma resposta com menor associação. Os autores avaliaram o nível de conhecimento dos universitários dos cursos de odontologia e enfermagem da universidade do Estado do Rio Grande do Norte frente ao câncer oral, e obtiveram um percentual relevante das respostas em relação ao tabaco. Já o álcool foi pouco referido. Canevese et al. ${ }^{20}$, ao utilizarem o questionário sem adaptações ou modificações, encontraram essa associação como a mais citada no fator de risco, corroborando nossos achados.

No presente estudo, não há dúvidas de que o hábito de fumar e o fumo passivo sejam prejudiciais à saúde. $\mathrm{O}$ mesmo foi encontrado por Canevese et al. ${ }^{20}$, que avaliaram o conhecimento dos usuários de uma unidade básica de saúde no Rio Grande do Sul sobre câncer de boca, utilizando o questionário de Rodrigues ${ }^{21}$, pela teoria da resposta ao item, e os participantes foram unânimes em dizer que o fumo é prejudicial à saúde; porém, alguns afirmam que fumar perto de outras pessoas não seria prejudicial.

Ainda que esteja bem estabelecido o fato de a alta ingestão de legumes frescos, frutas, peixes e frutos ser preventiva contra o câncer bucal ${ }^{12,20}$, os achados do presente estudo corroboram os estudos que utilizaram o questionário elaborado pela teoria de resposta ao item. Canevese et al. ${ }^{20}$ observaram que houve indivíduos que não creditam uma dieta saudável a um fator de prevenção para a doença. Entretanto, Rodrigues ${ }^{21}$ verificou que a maioria dos estudantes questionados acreditava que a alimentação pudesse contribuir para a prevenção do câncer de boca.

Quando questionados a respeito dos prejuízos que esse tipo de câncer pode trazer aos pacientes, a maior parte dos universitários concordou sobre as possíveis limitações, bem como com o fato de que inicialmente não causa dor. Esses resultados vão ao encontro aos achados por Souza e Carvalho ${ }^{22}$, ao dizerem que, em geral, o câncer de boca é assintomático, sendo que, em casos mais avançados, a dor possa existir. Outra complicação frequentemente relatada é a desnutrição relacionada ao $\mathrm{CCO}^{23}$, que, por vezes, pode estar relacionada ao trismo, o qual pode afetar a fala e a voz e prejudicar a mastigação e a deglutição ${ }^{24,25}$.

Os universitários entrevistados narraram que procurariam um médico ou dentista para a solução de alteraçóes na boca com mais de 15 dias. Segundo estudo de Cruz-Moreira ${ }^{25}$, 81,8\% dos estudantes avaliados em uma universidade católica do Equador nunca realizaram triagem para o câncer bucal. Silva ${ }^{26}$ acrescenta que cabe aos profissionais realizarem um exame clínico detalhado para reconhecer e tratar as lesóes, e conhecerem os exames complementares para um diagnóstico. Os encaminhamentos tardios são frequentes e são em razão do conhecimento e das habilidades inadequadas desses profissionais $^{27,28}$.

Riveira ${ }^{29}$ enfatiza que o conhecimento dos profissionais de saúde a respeito do $\mathrm{CCO}$ pode influenciar significativamente no declínio das taxas de incidência desse tipo de câncer nas próximas décadas. Um estudo de coorte, que teve o objetivo de analisar a sobrevida específica de cinco anos e fatores associados ao câncer de boca no Brasil, observou que essa taxa (entre 2002 e 2003) foi de $60 \%{ }^{30}$. Corroborando esses achados, os estudos de Canevese et al. ${ }^{20}$ e Rodrigues ${ }^{21}$ destacam que os dois estudos utilizaram o mesmo questionário para esse tipo de câncer e que tem cura, mas precisa de atenção e acompanhamento.

Ainda que o câncer oral não seja o mais prevalente, tem um prognóstico de mortalidade e principalmente de morbidade nos pacientes, essencialmente quando descoberto tardiamente após o aparecimento dos sintomas ${ }^{12,28}$. O diagnóstico tardio, geralmente quando os tumores já estão mais profundos, possui a probabilidade de ter o estágio mais avançado da doença. Nesses casos, comumente, os pacientes recebem o tratamento com cirurgia e radioterapia ${ }^{28}$, conforme os universitários demonstraram conhecimento, embora Canevese et al. ${ }^{20}$ tenham revelado que os indivíduos estudados demonstram não saber sobre a associação da cirurgia com os outros tratamentos.

No estudo de Domingos et al. ${ }^{7}$, foi aplicado um questionário para estudantes do curso de enfermagem e odontologia quanto ao conhecimento sobre o câncer de boca; nele, verificou-se diferença significativa entre estudantes no período inicial, intermediário e final da graduação, e os estudantes do período final demonstraram menor conhecimento, diferentemente do estudo no qual os universitários das fases finais retrataram maior conhecimento sobre o assunto. Ainda no estudo de Domingos et al. ${ }^{7}$, os estudantes foram questionados sobre a referência do conhecimento adquirido, e a maior parte relatou a graduação, seguida de propagandas de TV ou Internet e livros, revistas, artigos ou jornais. 
Canevese et al..$^{20}$ mostraram que o conhecimento dos usuários de uma unidade básica de saúde é inadequado quanto ao reconhecimento de alguns fatores de risco, características, prevalência, faixa etária e incidência. Nesse contexto, as universidades, como instituiçóes sociais, e os cursos da área da saúde devem preparar profissionais com habilidades para atuar na resolução das necessidades de saúde pública ${ }^{7}$, bem como é indispensável incrementar açôes de saúde para combater, prevenir e melhorar os indicadores de sobrevida ${ }^{9,30}$, assim como reduzir a ocorrência de novos casos, ajudar no diagnóstico precoce e evitar a sobrecarga do sistema de saúde, fazendo com que os recursos destinados ao tratamento e ao acompanhamento de pacientes oncológicos não sejam insuficientes ${ }^{31}$.

Uma das limitaçôes foi a necessidade de o e-mail ser enviado somente pelas coordenações dos cursos, o que pode ter implicado em uma baixa adesão; outra limitação foi a própria baixa adesão dos estudantes para responderem ao questionário, o que pode reduzir a validade externa do estudo.

\section{CONCLUSÃO}

Em conclusão, a maior parte dos universitários da Unisul tem conhecimento sobre o CCO. No entanto, sugere-se o aprofundamento sobre o conhecimento dessa enfermidade.

\section{CONTRIBUIÇÕES}

Todos os autores participaram de todas as etapas do manuscrito e aprovação da versão final.

\section{DECLARAÇÃO DE CONFLITO DE INTERESSES}

Nada a declarar.

\section{FONTES DE FINANCIAMENTO}

Não há.

\section{REFERÊNCIAS}

1. Instituto Nacional de Câncer José Alencar Gomes da Silva. Estimativa 2018: incidência de câncer no Brasil [Internet]. Rio de Janeiro: INCA; 2017. [acesso 2018 mar. 15]. Disponível em: http://www1.inca.gov.br/inca/ Arquivos/estimativa-2018.pdf

2. Campana IG, Goiato MC. Tumores de cabeça e pescoço: epidemiologia, fatores de risco, diagnóstico e tratamento. Rev. Odontológica de Araçatuba [Internet]. 2013 [acesso 2018 jan. 20];34(1):20-26. Disponível em: https:// repositorio.unesp.br/handle/11449/133244
3. Formigosa JAS, Costa LS, Vasconselos EV. Representaçôes sociais de pacientes com câncer de cabeça e pescoço frente à alteraçáo da imagem corporal. J Res: Fundam Care Online. 2018;10(1):180-189. doi: http://dx.doi. org/10.9789/2175-5361.2018.v10i1.180-189

4. Ferlay J, Soerjomataram I, Ervik M, et al., editors GLOBOCAN 2012: Estimated Cancer Incidence, Mortality and Prevalence Worldwide in 2012 v1.0 [Internet]. Lyon, France: International Agency for Research on Cancer; 2013 [cited 2018 Mar 15]. (IARC CancerBase N. 11) Available from: http://globocan.iarc.fr

5. Freitas RM, Rodrigues AMX, Júnior Matos AF, et al. Fatores de risco e principais alteraçôes citopatológicas do câncer bucal: uma revisão de literatura. RBAC [Internet]. 2016 [acesso 2018 mar. 18];48(1):13-18. Disponível em: http://www.rbac.org.br/artigos/fatores-de-risco-eprincipais-alteracoes-citopatologicas-do-cancer-bucaluma-revisao-de-literatura/

6. Oliveira JMB, Pinto LO, Lima NGM, et al. Câncer de boca: avaliação do conhecimento de acadêmicos de odontologia e enfermagem quanto aos fatores de risco e procedimentos de diagnóstico. Rev Bras Cancerol [Internet]. 2013 [acesso 2018 mar. 15];59(2):211-218. Disponível em: http://www1. inca.gov.br/rbc/n_59/v02/pdf/08-cancer-de-bocaavaliacao-do-conhecimento-de-academicos-deodontologia-e-enfermagem-quanto-aos-fatores-derisco-e-procedimentos-de-diagnostico.pdf

7. Domingos PAS, Passalacqua MLC, Oliveira ALBM, Câncer bucal: um problema de saúde pública. Rev Odontol Univ Cid São Paulo. 2014;26(1):46-52. doi: https://doi.org/10.26843/ro_unicid.v26i1.285

8. Perea LME, Peres MA, Boing AF, et al. Tendência de mortalidade por câncer de boca e faringe no Brasil no período 2002-2013. Rev Saúde Pública, 2018;52:10. doi: http://dx.doi.org/10.11606/s15188787.2018052000251

9. Goldemberg DC, Araujo LHL, Antunes HS, et al. Tongue cancer epidemiology in Brazil: incidence, morbidity and mortality. Head Neck. 2018;40(8):18341844. doi: http://dx.doi.org/10.1002/hed.25166

10. Weatherspoon DJ, Chattopadhyay A, Boroumand S, et al. Oral cavity and oropharyngeal cancer incidence trends and disparities in the United States: 2000-2010. Cancer Epidemiol. 2015 Aug;39(4):497-504. doi: https://doi. org/10.1016/j.canep.2015.04.007

11. Chen F, Yan L, Lin L, et al. Dietary score and the risk of oral cancer: a case-control study in southeast China. Oncotarget. 2017;8(21)34610-34616. doi: https://doi. org/10.18632/oncotarget.16659

12. Dhanuthai K, Rojanawatsirivej $S$, Thosaporn W, et al. Oral cancer: a multicenter study. Med Oral Patol Oral Cir Bucal. 2018;23(1):e23-e29. doi: http://dx.doi. org/10.4317/medoral.21999 
13. Tarvainen L, Suojanen J, Kyyronen P, et al. Occupational risk for oral cancer in Nordic Countries. Anticancer Res. 2017;37(6):3221-3228. doi: http://dx.doi. org/10.21873/anticanres.11684

14. Owosho AA, Wiley R, Stansbury T, et al. Trends in human papillomavirus-related oropharyngeal squamous cell carcinoma incidence, Vermont 1999-2013. J Community Health. 2018;43(4):731-737. doi: https:// doi.org/10.1007/s10900-018-0477-1

15. Moro JS, Maroneze MC, Ardenghi TM, et al. Oral and oropharyngeal cancer: epidemiology and survival analysis. Einstein, 2018;16(2):eAO4248. doi: https:// doi.org/10.1590/S1679-45082018AO4248

16. Zumsteg ZS, Cook-Wiens G, Yoshida E, et al. Incidence of oropharyngeal cancer among elderly patients in the United States. JAMA Oncol. 2016;2(12):1617-1623. doi: https://doi.org/10.1001/jamaoncol.2016.1804

17. Chotipanich A, Siriarechakul S, Mungkung OO. Role of high-risk human papillomavirus in the etiology of oral and oropharyngeal cancers in Thailand: a case-control study. SAGE Open Med. 2018;6:1-8. doi: https://doi. org/10.1177 / 2050312118765604

18. Bezerra NVF, Leite KLF, Medeiros MMD, et al. Impact of the anatomical location, alcoholism and smoking on the prevalence of advanced oral cancer in Brazil. Med Oral Patol Oral Cir Bucal. 2018;1;23(3):e295-301. doi: http://dx.doi.org/doi:10.4317/medoral.22318

19. Peters TM, Phillips C, Murrab VA. Is oral biopsy associated with change in tobacco or alcohol use? J Oral Maxillofac Surg. 2017;75(10): 2117-2126. doi: http:// dx.doi.org/10.1016/j.joms.2017.03.019

20. Canevese TR, Cericato GO. Conhecimento dos usuários de uma unidade básica de saúde sobre câncer bucal na cidade de Mato Castelhano, Rio Grande do Sul. J Oral Invest 2015; 4(1):44-51. doi: https:// doi.org/10.18256/2238-510X/j.oralinvestigations. v4n1p44-51

21. Rodrigues MAB. Elaboração, padronização e aplicação de questionário para avaliação de conhecimento sobre câncer bucal validado pela teoria de resposta ao item [dissertação na Internet]. Araçatuba (SP): Universidade Estadual Paulista, Faculdade de Odontologia de Araçatuba, Curso de Odontologia; 2011. [acesso 2018 jun. 2]. 88f. Disponível em: http://hdl.handle.net/11449/95412

22. Souza AL, Carvalho CHP. Nível de conhecimento da população e dos odontólogos no sertão paraibano sobre o câncer oral. RSC online [Internet] 2017 [acesso 2018 jun. 2];6(1):5-19. Disponível em: http://www.ufcg.edu. br/revistasaudeeciencia/index.php/RSC-UFCG/article/ view/376/268

23. Marques RSO, Costa AS, Peixoto ARA. Qualidade de vida em deglutição e câncer de cabeça e pescoço: revisão de literatura. Rev Bahiana Odontol. 2017;8(1):26-32. doi: http://dx.doi.org/10.17267/2596-3368dentistry. v8i1. 1260
24. Marrafon CS, Matos LL, Zenari MS, et al. Programa terapêutico fonoaudiológico para abertura de boca em pacientes com câncer de boca e orofaringe em radioterapia adjuvante: estudo piloto. CoDAS. 2018;30(2) e20160221. doi: http://dx.doi.org/10.1590/2317$1782 / 20182016221$

25. Cruz-Moreira K, Huamán-Garaicoa F, Mena G. Knowledge of oral cancer among the community served during the stomatological lesion prevention campaign conducted at Universidad Católica de Santiago de Guayaquil - Ecuador. Acta Odontol Latinoam [Internet]. 2017 [acesso 2018 jun. 9];30(3):113-117. Disponível em: http://www.scielo.org.ar/pdf/aol/v30n3/v30n3a04.pdf.

26. Silva BLR, Silva Neto MS, França DCC, et al. Perfil dos participantes do programa permanente de prevenção e diagnóstico precoce das doenças bucais, com ênfase no câncer de boca, no município de Cuiabá-MT. Arch Health Invest, 2017;6(3):141-144. doi: http://dx.doi. org/10.21270/archi.v6i3.1924

27. Varela-Centelles P, Insua A, Seoane-Romero JM, et al. Available web-based teaching resources for health care professionals on screening for oral cancer. Med Oral Patol Oral Cir Bucal. 2015;20(2):e144-9. doi: http://dx.doi. org/10.4317/medoral.20163

28. Guizard AVN, Dejardin OJ, Launay LC, Bara S, Lapôtre-Ledoux BM, Babin EB, Launoy GD, Ligier KA. Diagnosis and management of head and neck cancers in a high-incidence area in France: a population-based study. Medicine (Baltimore). 2017;96:26(e7285). doi: http:// dx.doi.org/10.1097/MD.0000000000007285

29. Riviera C. Essentials of oral cancer. Int J Clin Exp Pathol. 2015;8(9):11884-11894.

30. Bonfante GMS, Machado CJ, Souza PEA, et al. Sobrevida de cinco anos e fatores associados ao câncer de boca para pacientes em tratamento oncológico ambulatorial pelo Sistema Único de Saúde, Brasil. Cad Saúde Pública. 2014;30(5):983-997. doi: http://dx.doi. org/10.1590/0102-311X00182712

31. Castro SA. Levantamento das ocupaçóes relacionadas ao diagnóstico de câncer de cavidade oral e orofaringe no município de Curitiba de 1998 a 2012. [dissertação na Internet]. Curitiba (SC): Universidade Federal do Paraná, Curso de Odontologia; 2017. [acesso 2018 jun. 9]. 84f. Disponível em: http://hdl.handle.net/1884/49042.

Recebido em 2/5/2019 Aprovado em 2/8/2019 